\title{
A Comparison between the hp-version of Finite Element Method with EIDORS for Electrical Impedance Tomography
}

\author{
N. Saeedizadeh ${ }^{1}$; S. Kermani ${ }^{1}$; H. Rabbani ${ }^{1}$
}

\begin{abstract}
In this study, a hp-version of Finite Element Method (FEM) was applied for forward modeling in image reconstruction of Electrical Impedance Tomography (EIT). The EIT forward solver is normally based on the conventional Finite Element Method (h-FEM). In h-FEM, the polynomial order (p) of the element shape functions is constant and the element size (h) is decreasing. To have an accurate simulation with the h-FEM, a mesh with large number of nodes and elements is usually needed. In order to overcome this problem, the high order finite element method (p-FEM) was proposed. In the p-version, the polynomial order is increasing and the mesh size is constant. Combining the advantages of two previously mentioned methods, the element size (h) was decreased and the polynomial order (p) was increased, simultaneously, which is called the hpversion of Finite Element Method (hp-FEM). The hp-FEM needs a smaller number of nodes and consequently, less computational time and less memory to achieve the same or even better accuracy than hFEM. The SNR value is $42 \mathrm{db}$ for hp-FEM and is $9 \mathrm{db}$ for $\mathrm{h}$-FEM. The numerical results are presented and verified that the performance of the hp-version is better than of the h-version in image reconstruction of EIT.
\end{abstract}

\section{KEYWORDS}

Electrical Impedance Tomography (EIT), Finite Element Method (FEM), Conventional Finite Element Method (h-FEM), High order Finite Element Method (p-FEM), hp-FEM, EIDORS.

\section{INTRODUCTION}

The Electrical Impedance Tomography (EIT) is to reconstruct the unknown conductivity distribution in an object $\Omega$ by measuring the low-frequency electrical potentials on the boundary. A set of contact electrodes is attached to the boundary $\partial \Omega$ and different current patterns are applied to the electrodes in order to measure the surface potentials. The conductivity distribution is to be reconstructed from these potential measurements. The applications of EIT in medical, detection of cancerous tumors from breast tissue [1]-[3], measuring brain function [4]-[5], and gastric functions [6]-[8]. In industry EIT has applications such as imaging of fluid flows in process pipelines [9], and non-destructive testing of materials [10]. For a review on EIT see also [6],[9].

EIT is a badly posed inverse problem. Small errors in the measurements or in solution of the forward problems can produce large errors in the reconstruction. One of the important approaches to solve forward problem is the finite element approximation. Usually this method is used with complete electrode methods because this model predicts the measured data with more accuracy than continuum, gap and shunt models. The conventional Finite Element Method (hFEM) and the high-order Finite Element Method (p-FEM) are considered to solve the forward problems. In the $\mathrm{h}$ FEM, the polynomial order (p) of the element shape functions is constant and the element size (h) is decreasing. With decreasing the element size, discretisation error is reduced. In the p-version, the basis functions are higher order Legendre polynomials. In this method the polynomial order is increased and the mesh size is constant. Combining the advantages of two previously mentioned methods, the

\footnotetext{
${ }^{1}$ Department of Medical Physics and Medical Engineering, Isfahan University of Medical Sciences, Isfahan, Iran (email: nargessaidi@gmail.com) (email: kermani@med.mui.ac.ir) (email: $\underline{\text { h_rabbani@med.mui.ac.ir }}$ )
} 
element size (h) was decreased and the polynomial order (p) was increased, simultaneously, which is called the hpversion of Finite Element Method (hp-FEM) [25]-[27]. For more detail about h-, p- and hp-versions of FEM See a book by Szabo and Babuska [10]. In this paper, the hpFEM was used for EIT problem. The high order FEM is capable of achieving even better accuracy than conventional FEM.

The following contributions are made here:

- Using a finer mesh near the electrodes because the electric potential changes more rapidly in these areas [11].

- Improving the algorithm efficiency by optimizing the number of mesh elements [18].

- Considering and carrying out simulations on the h- and p- version of FEM [12].

- Using a EIDORS (Electrical Impedance and Diffuse Optical Reconstruction Software) toolkit developed for MATLAB, for mesh generation, reconstruction and displaying the reconstructed images [13]-[17].

- The implementation is performed with PDE Toolbox in MATLAB Software (C) and EIDORS.

The novelty of this paper can be classified as:

- Refinement of the mesh to achieve stable voltage in PDE Toolbox of MATLAB and using this result as precise solution.

- Comparison between the results of the reconstructed image obtained by hp-FEM and EIDORS.

In section 2, the high order FEM forward model was described. In section 3 , results of applying the implemented method on a 2-D model are shown. Some details of the presented method were discussed in section 4.

\section{METHOD AND MATERIAL}

\section{A. High Order FEM of the Complete Electrode Model}

The forward problem involves the solution of a Maxwell equation. In the quasi-static and low frequencies approximation the fields can be described with Laplace equation.

$$
\nabla .(\sigma \nabla u)=0 \quad \text { in } \Omega
$$

Here, $\sigma$ and $\mathrm{u}$ denoted the conductivity and electric potential distribution.

The complete electrode model with the mixed boundary condition (Dirichlet and Neumann)

$V_{l}=u+Z_{l} \sigma \frac{\partial u}{\partial n} \quad$ on $E_{l} \quad 1 \leq l \leq L$

$I_{l}=\int_{E_{l}} \sigma \frac{\partial u}{\partial n} d s \quad$ on $E_{l} \quad 1 \leq l \leq L$

$\sigma \frac{\partial u}{\partial n}=0 \quad$ on $\partial \Omega / \bigcup_{l=1}^{L} E_{l}$

Where $I_{l}$ current injected from the $l$ th electrode, $Z_{l}$ is the contact impedances between $l$ th electrode and object and $V_{l}$ is the voltage measured on electrode $E_{l}$. In order to make sure that the model has a unique solution, Kirchhoff's current and voltage laws $\left(\sum_{l=1}^{L} I_{l}=0\right.$ and $\left.\sum_{l=1}^{L} V_{l}=0\right)$ must be satisfied.

The Finite Element solution of equations (1) is the:

$$
u_{F E}=\sum_{i=1}^{N} \alpha_{i} \varphi_{i}
$$

Where $\mathrm{N}$ the number of nodes, $\varphi_{i}$ the basis functions and the voltage vector on electrodes is represented as:

$$
\begin{aligned}
& U_{F E}=\sum_{j=1}^{L-1} \beta_{j} e_{j} \\
& e_{1}=(1,-1,0,0, \ldots, 0)^{T} \\
& e_{2}=(0,1,-1,0, \ldots, 0)^{T} \\
& \cdot \\
& \cdot \\
& e_{l}=(0,0,0, \ldots, 1,-1)^{T}
\end{aligned}
$$

By solving the linear system of equations $A \lambda=f$, the coefficients $\alpha_{i}$ and $\beta_{j}$ can be found. In this equation

$$
A=\left[\begin{array}{cc}
B & C \\
C^{T} & G
\end{array}\right], \lambda=\left[\begin{array}{c}
\alpha_{i} \\
\beta_{j}
\end{array}\right], f=\left[\begin{array}{l}
0 \\
I
\end{array}\right]
$$




$$
\begin{aligned}
& B_{e}(i, j)=\int_{\Omega} \nabla \varphi_{i} \nabla \varphi_{j} d x+\sum_{l=1}^{L} 1 / Z_{l} \int_{\partial \Omega} \varphi_{i} \varphi_{j} d s \\
& C_{e}(i, j)=-\int_{E_{l}} 1 / Z_{l} \varphi_{i} d s \\
& G_{e}(i, l)=1 / Z_{l}\left|E_{l}\right| \text { for } i=l
\end{aligned}
$$

Where indices $i=1, \ldots, N, l=1, \ldots, L$ and $\left|E_{l}\right|$ is the area of the $l$ th electrode.

The detail of numerical implementation of the forward problem based on CEM has been discussed in [19],[20].

\section{B. Hierarchic shape functions}

The set of one-dimensional hierarchic shape functions, introduced by Szabo and Babuska (1991), is given by

$$
\begin{aligned}
& \varphi_{1}(\varepsilon)=1 / 2(1-\varepsilon) \\
& \varphi_{2}(\varepsilon)=1 / 2(1+\varepsilon) \\
& \varphi_{n}(\varepsilon)=\phi_{(n-1)}(\varepsilon) \quad n=3,4, \ldots, p+1 \\
& \phi_{j}(\varepsilon)=\sqrt{\frac{2 j-1}{2}} \int_{-1}^{\varepsilon} L_{j-1}(x) d x \quad \quad j=2,3, \ldots \\
& =\frac{1}{\sqrt{4 j-1}}\left(L_{j}(\varepsilon)-L_{j-2}(\varepsilon)\right)
\end{aligned}
$$

Where $L_{j}(\varepsilon)$ are the Legendre polynomials.

$$
\mathrm{L}_{\mathrm{j}}(x)=\frac{1}{2^{j} j !} \frac{d^{j}}{d x^{j}}\left(x^{2}-1\right)^{j} . \quad j=0,1, \ldots
$$

The first, two shape functions $\varphi_{1}(\varepsilon), \varphi_{2}(\varepsilon)$ are called nodal shape functions or nodal modes. The functions $\varphi_{n}(\varepsilon), \mathrm{n}=3,4 \ldots$ are called internal shape functions, internal modes, or bubble modes [13][24].

In 2D, the standard triangular element, shown in Fig.1. In this figure:

$\mathrm{P}_{1}=\frac{1}{2}\left(1-\varepsilon_{t}-\frac{\eta_{t}}{\sqrt{3}}\right)$

$\mathrm{P}_{2}=\frac{1}{2}\left(1+\varepsilon_{t}-\frac{\eta_{t}}{\sqrt{3}}\right)$

$\mathrm{P}_{3}=\frac{\eta_{t}}{\sqrt{3}}$.

These functions are called barycentric triangular, or area coordinates.

$$
\mathrm{P}_{1}+\mathrm{P}_{2}+\mathrm{P}_{3}=1
$$

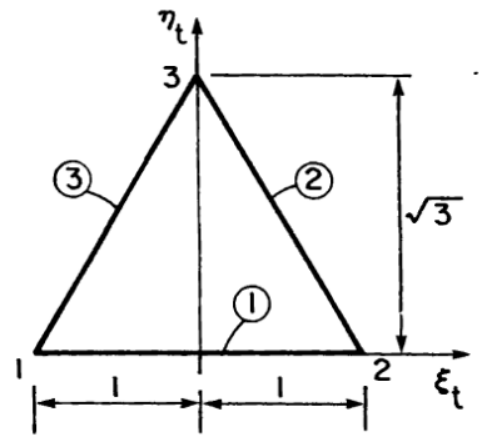

Figure 1: The standard triangular element [13].

Hierarchic shape functions for the standard triangular element are defined as:

- Nodal shape functions: These shape functions are the same as the shape functions in h-FEM.

- Side modes: There are $3(\mathrm{p}-1)$ side modes for each triangle. The side modes associated with side 1 are:

$\varphi_{\mathrm{i}}^{(1)}=P_{1} P_{2} \phi_{i}\left(P_{2}-P_{1}\right)$

The definition of the other side modes is analogous.

- Internal mode: the first internal mode defined as:

$$
\varphi_{1}^{(0)}=P_{1} P_{2} P_{3}
$$

Other internal mode generated by multiplying $\varphi_{1}^{(0)}$ by Legendre polynomial and products of Legendre polynomials.

\section{Numerical Experiments}

In order to apply the p-FEM to the EIT forward problem, the physical domain is first divided into triangular elements. Each of the triangular elements in Cartesian coordinates $(x, y)$ is mapped into a local reference element in $(\varepsilon, \eta)$ coordinates. The global matrix in (8) is assembled by going through each triangular element and by computing the elemental matrices $B_{e}, C_{e}$ and $G_{e}$ in local reference coordinates. Note that the boundary contribution in (9) is nonzero only for elements on the outer boundary. For implementation of the hp-FEM on the EIT, the mesh is refined. In the next step, the hierarchic shape function is applied to achieve the final results. 


\section{RESUlt AND DiscuSSION}

A. Comparison between high order FEM and conventional FEM

A numerical experiment is set up to compare the efficiency of higher order FEM and conventional FEM. The meshes for h-FEM will have the same number of nodes as the hp-FEM. In this paper, the domain $\Omega$ is the unit circle with 16 electrodes attached on its boundary. The mesh used in the PDE Toolbox has 1136 triangular cells and 633 nodes. During the simulation, the contact impedances of the electrodes are set to one. The current patterns are designed in a way that 16 adjacent pairs are allocates as source and sink electrodes and one extra electrode is embedded as the ground electrode. Altogether, there are 16 current patterns and each current pattern has 13 voltage measurements on the electrodes. Therefore, the $16 * 13=208$ voltage is measured. To visualize the voltage on electrodes for all current patterns, all the 208 voltage values is stacked together. For obtaining a precise solution, refinement of the mesh in PDE Toolbox of MATLAB. The mesh used in precise solution has 290416 cells and 146433 nodes.

To compare the different forward solutions, in addition to visual inspection of the results, the signal-to-noise ratio (SNR) is computed, defined as:

$$
S N R=10 \log \left(\frac{\sum_{i=1}^{L}\left(V_{\text {exact }}(i)\right)^{2}}{\sum_{i=1}^{L}\left(V_{\text {numerical }}(i)-V_{\text {exact }}(i)\right)^{2}}\right)
$$

On the plots, the value of this error is reported in term of average error, where

$$
\text { AverageError }=1 / N \sum_{i=1}^{N}\left|\frac{V_{\text {numerica }}(i)-V_{\text {exact }}(i)}{V_{\text {exact }}(i)}\right|
$$

In Fig. 2 and Fig.3 the voltage values for first current pattern of h-FEM, hp-FEM and precise solution are shown. In Table.1, the SNR values for two methods (hFEM and hp-FEM) are compared. It can be seen that the hp-version is able to achieve better accuracy than the conventional FEM. In order to achieve an accuracy of just 0.002 the conventional FEM needs more than $10^{5}$ nodes, but the hp-FEM needs just 633 nodes.

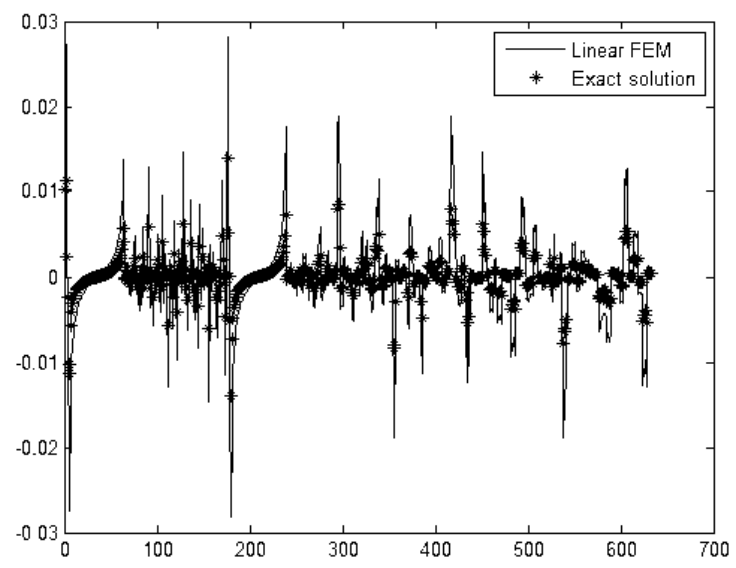

Figure 2: Results of the exact solution and h-FEM with the complete electrode model for first current pattern, Average Error $=1.81$.

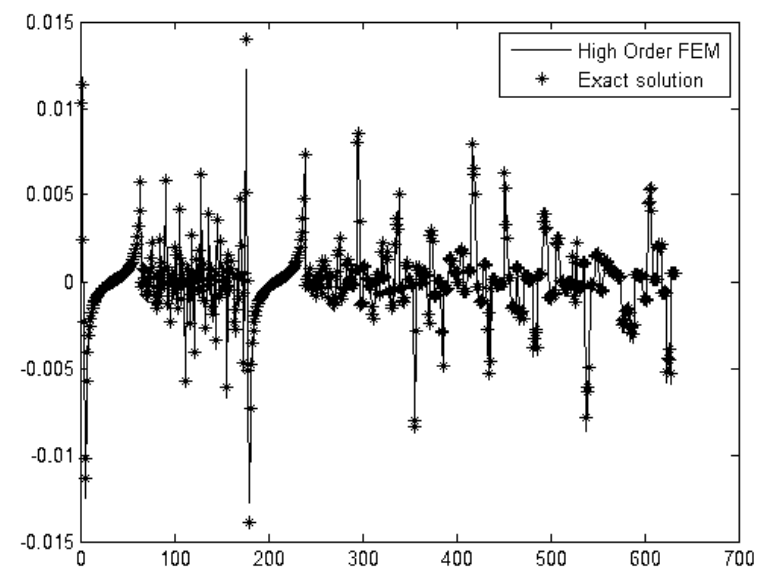

Figure 3: Results of the exact solution and hp-FEM with the complete electrode model for first current pattern, Average Error $=0.186$.

TABLE 1

SNR IN VOLTAGE COMPUTED WITH HP-FEM AND CONVENTIONAL FEM.

\begin{tabular}{l|l|}
\hline Parameter & SNR \\
\hline \hline hp-FEM & 42.5583 \\
\hline Conventional & 9.2237 \\
FEM & \\
\hline
\end{tabular}

According to these results, the hp-FEM is an ideal candidate for our problem. In [15], Pursiainen presented that performance of the p-version is better than that of the h-version. Our results-confirming the results of [15]revealed that $\mathrm{p}$ and $\mathrm{hp}$-version of finite element method provides more efficient tool for EIT forward modeling. 


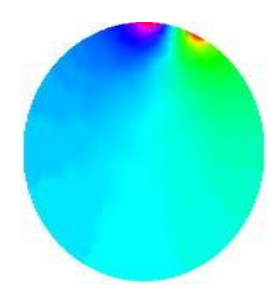

(a)
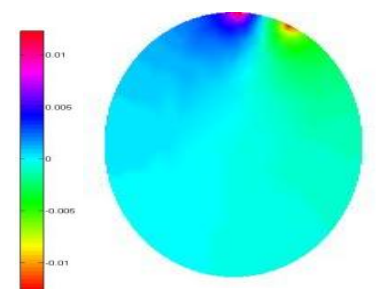

(b)
Figure 4: Computed voltage with hp-FEM (a) and precise solution (b) for first current pattern.

\section{B. Validation}

The main objective of EIT is reconstruction of images objects that are present in the applicator from the voltage data measured on the electrodes. The difference in voltage measurements between with and without the objects is defined as the secondary field. The secondary field is basically caused by the objects. So, it is essential that the forward model is able to simulate the secondary field accurately. To verify the accuracy of the high order FEM forward solver for the secondary field, we used as phantom shown in Fig.5.

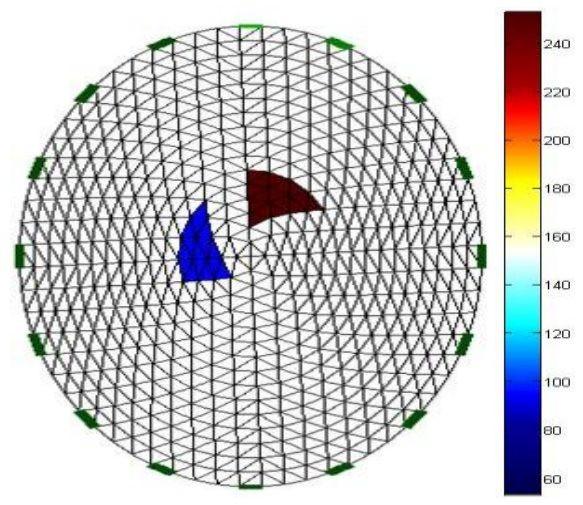

Figure 5: Triangular mesh used in EIDORS with two anomalies.

The experiment is carried out by computing the voltages on the electrodes without and with the object. The secondary field is simply the difference between the voltages computed with and without the object. The comparison between reconstructed image with hp-FEM and EIDORS is shown in Fig.6 and Table 2.

TABLE 2

Reconstructed image with proposed algorithm and EIDORS.

\begin{tabular}{l|l|}
\hline Parameter & SNR \\
\hline \hline hp-FEM & 45.7755 \\
\hline EIDORS & 42.451 \\
\hline
\end{tabular}

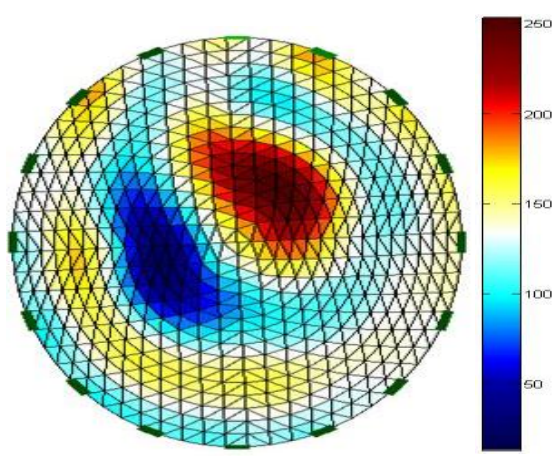

(a)

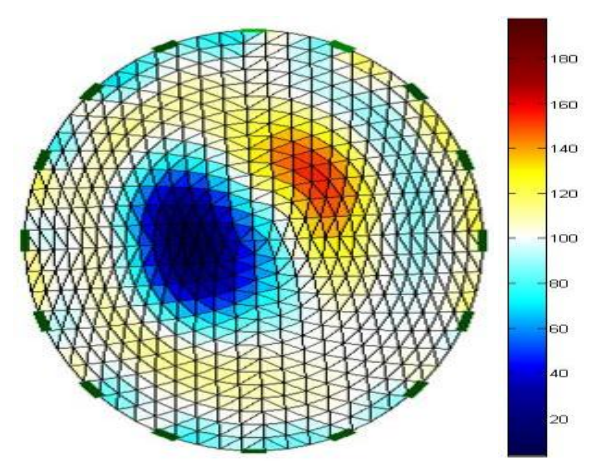

(b)

Figure 6: Reconstructed Image with hp-FEM (a) and EIDORS (b).

\section{CONCLUSION}

The high order finite element method has been developed and validated for the EIT forward problem. Numerical results show that hp-FEM is capable of achieving even better accuracy than conventional FEM. The total computational time and memory usage for hp-FEM are much less than the conventional FEM. The reconstructed image with hp-FEM has also been compared with EIDORS. In summary, the high order FEM is an efficient forward solver for Electrical Impedance Tomography.

\section{REFERENCES}

[1] L. Wang, W. Wang, T. Haung, B. Tanstall, "In-vitro Study of Electrical Characteristics of Human Female Breast Tissues," IEEE. Inter. Conf. on Electro/Information Tech, 2008.

[2] E. Demidenko, A. Borsic, Y. Wan, R. Halter, A. Hartov, "Statistical estimation of EIT electrode contact impedance using magic Toeplitz matrix," IEEE. Trans on Biomedical Eng, Vol. 58, Issue. 8, pp. 2194-2201, 2010.

[3] G. A. Ybarra, Q. H. Liu, G. Ye, K. H. Lim, J. H. Lee, W. T. Joines, and R. T. George, "Breast Imaging using Electrical Impedance Tomography (EIT)," CHAPTER. 15, American Scientific Publishers, 2007.

[4] N. Polydorides, W. R. B. Lionheard and H. McCann, "Krylov subspace iterative techniques: on the detection of brain activity 
with electrical impedance tomography," IEEE. Trans. on Medica Imaging, vol. 21, pp. 596-603, 2002.

[5] E. J. Woo, "Functional brain imaging using MREIT and EIT: Requirements and feasibility," 8th Int. Conf. on Bioelectromagnetism, pp. 131-134, 2011.

[6] P. W. A. Kunst, A. V. Noordegraaf, O. S. Hoekstra, P. E. Postmus, and P. M. J. Vries, "Ventilation and perfusion imaging by electrical impedance tomography: a comparison with radionuclide scanning," Physiology Meas, Vol. 19, pp. 481-490, 1998.

[7] I. Frerichs, G. Hahn and G. Hellige, "Thoracic electrical impedance tomographic measurements during volume controlled ventilationeffects of tidal volume and positive endexpiratory pressure," IEEE Trans Med Imaging, vol. 18, pp. 764-773, 2000.

[8] L. Zhangyong, X. Likun, W. Jinmin, L. Xiaoyan, G. Yijun, "Modeling the electrical characteristic of the stomach and reconstructing image," Int. Conf. on Computer Application and System Modeling, Vol. 6, pp. 220-224, 2010.

[9] S. Stanley, R. Mann and K. Primrose, "Tomographic imaging of fluid mixing in 3-d for single-feed semi-batch operation of a stirred vessel," Chemical Engineering Research and Design, Vol. 80, pp. 903-909, 2002.

[10] T. Vilhunen, L. M. Heikkinen, T. Savolainen, P. J. Vauhkonen, R. Lappalainen, J. P. Kaipio and M. Vauhkonen, "Detection of faults in resistive coatings with an impedance-tomography-related approach," Measurement Science and Technology, Vol. 13, pp. 865-872, 2002.

[11] Y. Zou and Z. Guo, "A review of electrical impedance techniques for breast cancer Detection," ELSEVIER, Medical Engineering \& Physics, Vol. 25, pp. 79-90, 2003.

[12] I. Frerichs, "Electrical impedance tomography in applications related to lung and ventilation: a review of experimental and clinical activities," Physiol. Meas, Vol. 21, pp. 1-21, 2000.

[13] B. Szabo and I. Babuska, "Finite Element Analysis," Interscience Publication. John Wiley \& Sons, New York, 1991.

[14] K. H. Lim, J. H. Lee, G. Ye and Q. H. Liu, "An Efficient Forward Solver in Electrical Impedance Tomography by Spectral Element Method," IEEE. Trans on Medical Imaging, Vol. 25, no. 8, 2006.

[15] S. Pursiainen and H. Hakula, "A High-order Finite Element Method for Electrical Impedance Tomography," Vol. 2, no. 3, pp. 260-264, 2006.

[16] A. Adler and W. Lionheart, "Uses and abuses of EIDORS: An extensible software base for EIT," Manchester Institute for Mathematical Sciences, online. http://www.manchester.ac.uk/mims/eprints, 2006, MIMS EPrint. 2008 .

[17] A. Adler, A. Borsic, N. Polydorides and W. Lionheart, "Simple FEMs aren't as good as we thought: experiences developing EIDORS v3.3," Manchester Institute for Mathematical Sciences, online. http://www.manchester.ac.uk/mims/eprints, 2008

[18] M. Vauhkonen, W. Lionhearty, L. Heikkinen, P. Vauhkonen and J Kaipio, "A MATLAB Package for the EIDORS project to reconstruct, two-dimensional EIT images," FIN-70211. Kuopio, Finland, 2000.

[19] N. Polydorides, "Image Reconstruction Algorithms for Soft-Field Tomography," Ph.D. dissertation, University of Manchester, Manchester, United Kingdom, 2002.

[20] W. Lionheart, "Developments in EIT reconstruction algorithms," 4th Workshop on Biomedical Applications of EIT, 2003.
[21] E. Burman and A. ERN, "Continuous Interior Penalty hp-Finite Element Methods for Advection and Advection-Diffiusion Equation," Mathematics of Computation, Vol. 76, no. 259, pp. 1119-1140, 2007.

[22] S. Jarvenpaa, "A finite element model for the inverse conductivity problem," Ph.D. dissertation, University of Helsinki, Finland, 1996.

[23] M. Vauhkonen, "Electrical Impedance Tomography and Prior Information," Ph.D. dissertation, University of Kuopio, Finland, 1997.

[24] B. Szabo, A. Duster and E. Rank, "The p-version of the Finite Element Method," Chapter. 5, Encyclopedia of Computational Mechanics, 2004.

[25] P.D. Ledger, "hp FEM: Efficient solvers, error estimation, adaptivity and accurate solutions," Computational. Eng, Swansea University, 2011.

[26] P. Houston, D. S. otzau and Th. P. Wihler, "Energy norm a posteriori error estimation of hp-adaptive Discontinuous Galerkin methods for elliptic problems," Tech. Report IMA Preprint Series 1985, Institute for Mathematics and its Applications, 2004.

[27] L. Zhu, S. Giani, P. Houston and D. S. Otzau "Energy norm APosteriori Error Estimation for hp-adaptive discontinuous Galerkin methods for elliptic problems in Three Dimentions," Mathematical Models and Methods in Applied Sciences (M3AS), online. http://www.worldscinet.com/m3as/m3as.shtml, 2011.

[28] D. S. Tawil, D. Rye and M. Velonaki, "Improved Image Reconstruction for an EIT-Based Sensitive Skin With Multiple Internal Electrodes," IEEE. Trans on robotics, Vol. 27, no. 3, 2011. 